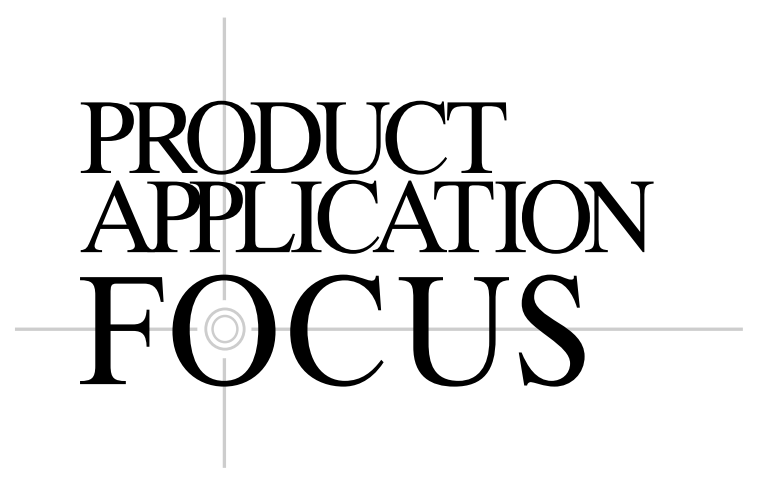

... a forum for manufacturers to describe the current and potential applications of new research instruments or products.

\title{
Detection of Mutations in Exon 8 of TP53 by Temperature Gradient 96-Capillary Array Electrophoresis
}

\author{
A. Torgunrud Kristensen, J. Bjørheim, M. Minarik¹, K.-E. Giercksky, \\ and P.O. Ekstrøm \\ The Norwegian Radium Hospital, Oslo, Norway, and ${ }^{1}$ Amersham \\ Biosciences, Sunnyvale, CA, USA
}

BioTechniques 33:650-653 (September 2002)

\begin{abstract}
Various capillary electrophoresis applications have increasingly been utilized in mutation detection. Separation of two species is either based on secondary structure or differences in melting of DNA due to the mutation. Detection of the mutant is based on its mobility difference in the sieving matrix. We have adapted a regular 96-capillary sequencing instrument, the MegaBACE ${ }^{T M} 1000$, for mutation detection based on thermodynamic stability and mobility shift during electrophoresis. Denaturation of the lower melting domain of the DNA was achieved with a gradually decreasing temperature gradient in combination with a chemical denaturant. Samples were analyzed for mutants in exon 8 of the TP53 gene from tumor samples and controls. Genomic DNA was PCR-amplified with one fluorescein labeled primer and one GC-clamped primer, diluted in water, and analyzed by temperature gradient 96-capillary array electrophoresis. Tumor samples and PCR reconstruction experiment samples were resolved by capillary gel electrophoresis under appropriate temperature gradient denaturing conditions. Ninety-six samples were analyzed in one run, with an analysis time of $30 \mathrm{~min}$ and a sensitivity to detect mutated alleles in wild-type background down to 0.4\%. The technique proved to be robust, in that the gradient compensates for temperature differences within the capillary chamber; thus, each capillary will pass through the optimal separating conditions around the theoretical melting temperature for TP53 exon 8, separating homoduplexes and heteroduplexes. This technique is applicable to any sequence previously analyzed by DNA melting gel techniques or sequences harboring iso-melting domains of 100-120 bp.
\end{abstract}

\section{INTRODUCTION}

Detection of DNA mutations in oncogenes and tumor suppressor genes is important in the evaluation of diagnosis and prognosis of cancer patients. Mutations in the TP53 tumorsuppressor gene are found at high frequency in a wide range of human cancers (12). TP53 codes for a protein that acts as a transcription factor and serves as a key regulator of the cell cycle. Inactivation of $\mathrm{p} 53$ by mutations disrupts the cell cycle and may lead to tumor formation (15). The majority of TP53 mutations are reported to span a $2-\mathrm{kbp}$ region of the gene that includes exons 5-8 (12). Different mutations in this gene have been related to treatment response in cancer patients $(4,9)$. A simple way to detect mutations within the gene would be of importance to large groups of cancer patients.

To establish an automated, high-throughput method for the detection of mutations with high sensitivity and robustness, the principles of melting gel techniques introduced by Fischer and Lerman (7) were adapted. The separation principle of melting gel techniques such as denaturant gradient gel electrophoresis (DGGE) (8), constant denaturant gel electrophoresis (CDGE) (13), temporal temperature gel electrophoresis (TTGE) (3), and constant denaturant capillary electrophoresis (CDCE) (14) are based on the melting behavior of the DNA double helix of a given fragment. This melting behavior is sequence-dependent. The melting of a domain in the DNA fragment will be detected as a reduction in DNA fragment mobility as it moves through the gel as a conse- 
quence of partial strand separation. The thermodynamics of the transition of DNA double strand to DNA single strand have been described by Fischer and Lerman (7), and computer programs for the analysis of denaturation are available (Medprobe, Oslo, Norway). Recent reports have demonstrated automation of melting gel theory by applying CDCE to an ABI 310 Genetic Analyzer for mutation detection in KRAS exon 1 and TP53 exons 5-8 (1,2). The ABI 310 is a single capillary instrument, thus limiting the throughput potential in mutation detection, whereas temperature gradient 96-capillary array electrophoresis combines the melting gel theory and automation in a 96- array format. Here we analyze PCR products from TP53 exon 8 with temperature gradient 96capillary array electrophoresis to evaluate the sensitivity, robustness, and throughput of the method.

\section{MATERIALS AND METHODS}

\section{Instrumentation}

The instrumentation was a standard multi capillary DNA sequencing instrument MegaBACE ${ }^{\mathrm{TM}} 1000$ DNA Analysis System (Amersham Biosciences, Oslo, Norway). The instrument is a high-throughput, fluorescence-based DNA system utilizing capillary electrophoresis with up to 96 capillaries operating in parallel. Coated fused silica capillaries were obtained from the instrument's manufacturer in sets of 16 capillaries, thus giving the possibility to run 16, 32, 48, 64, 80 and 96 , respectively. The capillaries have an o.d. of $200 \mu \mathrm{m}$ and an i.d. of $75 \mu \mathrm{m}$. The distance from the cathode to the detector is $40 \mathrm{~cm}$. The MegaBACE platform uses confocal laser scanning focused on each capillary.

\section{Temperature Gradient 96-Capillary Array Electrophoresis}

Regular 3\% linear polyacrylamide matrix containing $7 \mathrm{M}$ urea was replaced in capillaries before every run, by applying high-pressure nitrogen. PCR products were diluted 1:50 in water before electrokinetic injection, accomplished by applying $10 \mathrm{kV}$ for $12 \mathrm{~s}$. The electrophoretic condition was constant field of $150 \mathrm{~V} / \mathrm{cm}$. The partially melting of DNA fragments was achieved by a combination of urea and temperature surrounding the capillaries. The separating temperature used was a gradient going from $54^{\circ} \mathrm{C}$ down to $52^{\circ} \mathrm{C}$ in step of $0.5^{\circ} \mathrm{C}$. Each temperature was held for $210 \mathrm{~s}$. The gradient was followed by constant temperature at $50^{\circ} \mathrm{C}$ in $60 \mathrm{~min}$.

\section{Samples}

Tumor tissue from patients with colorectal carcinoma was immediately frozen after removal and stored at $-70^{\circ} \mathrm{C}$. DNA was extracted from tumor tissue and control samples with QIAamp ${ }^{\circledR}$ DNA Kit (Qiagen, Valencia, CA, USA). Sensitivity reconstruction experiments were performed by serial dilution of mutated alleles with wild-type alleles, followed by formation of heteroduplexes by boiling and re-annealing. With the use of computer software, we measured the peak areas presented in the electropherograms. The areas under the peaks were used as a quantitative measurement of the mutant fraction in the samples. Mutant fractions were calculated as previously described (6).

\section{PCR}

Fragment exon 8 of the TP53 gene was PCR-amplified with one 60-mer and one 20-mer primer (MedProbe): $5^{\prime}$ Fluorescein-ATCCTGAGTAGTGGTAATCT-3' and 5'-CGCCCGCCGCGCCCCGCGCCCGTCCCGCCGCCCCCGCCCGTACCTCGCTTAGTGCTCCCT- 3 '. The $25-\mu \mathrm{L}$ reaction was performed using $0.06 \mathrm{U} / \mu \mathrm{L}$ Taq DNA polymerase (ABgene, Epsom,UK) with corresponding buffer. The magnesium, deoxynucleotide, and primer concentration was 2.5 $\mathrm{mM} \mathrm{MgCl} 2,0.32 \mathrm{mM}$ dNTP (ABgene), and $0.05 \mathrm{mM}$ each primer, respectively. Amplifications were performed using the PTC-200 ${ }^{\mathrm{TM}}$ thermal cycler (MJ Research, Waltham, MA, USA), using the following cycling conditions: $120 \mathrm{~s}$ at $94^{\circ} \mathrm{C}$, 35 cycles of $30 \mathrm{~s}$ at $94^{\circ} \mathrm{C}, 30 \mathrm{~s}$ at $55^{\circ} \mathrm{C}$, and $60 \mathrm{~s}$ at $72^{\circ} \mathrm{C}$. Formation of heteroduplexes between wild-type and mutation was achieved by heating the PCR product to $94^{\circ} \mathrm{C}$ for $3 \mathrm{~min}$ and allowing re-annealing at $65^{\circ} \mathrm{C}$ for $30 \mathrm{~min}$.

\section{RESULTS}

By gradually decreasing the temperature in combination with a chemical denaturant in the gel, separation between homoduplexes and heteroduplexes in mutated TP53 exon 8 was achieved. Several different temperature gradients were used to analyze TP53 exon 8 . The best separation of homoduplexes and heteroduplexes was achieved by decreasing the temperature in $0.5^{\circ} \mathrm{C}$ increments from $54^{\circ} \mathrm{C}$ down to $52^{\circ} \mathrm{C}$. All the mutations we have analyzed so far have displayed a distinct peak pattern with regard to homoduplexes and heteroduplexes.

PCR product from a mutated sample was analyzed in all 96 capillaries simultaneously. Figure 1 depicts 16 representative electropherograms from all arrays within the instrument. The electropherograms are not normalized or corrected for differences in running conditions because of current differences between the capillaries. All electropherograms displayed baseline separation between wild-type and mutant homoduplexes and heteroduplexes. The analysis time for 96 samples was $30 \mathrm{~min}$.

Mutant and wild-type PCR products were mixed in various ratios in the sensitivity reconstruction experiment. Figure 2 demonstrates loss of heteroduplex signal below mutant fraction of $4 \times 10^{-3}$. This is in accordance with previously reported capillary DNA melting techniques.

Figure 3 demonstrates the robustness of the temperature gradient with respect to the separation of different mutations occurring in exon 8 of the TP53 gene. The mutations displayed are sited within $48 \mathrm{bp}$ of the conservative region of the gene. The figure depicts six of the 12 possible combinations of base substitutions. The mutations are distributed from codon 266 to codon 282 . Three different mutations in the hot spot codon 273 are shown.

\section{DISCUSSION}

In this study, we have adapted a multiple capillary sequencing instrument with laser-induced fluorescence for detection of mutations in exon 8 of the TP53 gene. Alteration of a single base in the low melting domain of the fragment to be analyzed will alter the melting behavior of most mutant homoduplexes and all mutant/wild-type heteroduplexes, respectively. Each 
mutation will consequently reveal a specific peak (homoduplex) or peaks (heteroduplexes) in the electropherogram depending on whether or not the mutants have been completely transformed into heteroduplexes by excess wild-type DNA. Mutant separation was achieved by a combination of chemical denaturant and temperature control in the capillary. Selection of temperature gradients was based on theoretical melting behavior. We used the theoretical melting temperature of the fragment, $76^{\circ} \mathrm{C}$, corrected for chemical denaturant $(7 \mathrm{M}$ urea reducing the melting temperature by approximately $21^{\circ} \mathrm{C}$ ). The first gradient run on the instrument was $56^{\circ} \mathrm{C}-54^{\circ} \mathrm{C}$. This gradient revealed melting of the DNA at too high temperature (data not shown). This can be explained by a difference of the temperature control of the MegaBACE compared to the true temperature in the capillary chamber. Consequently, the starting point of the temperature gradient was lowered until satisfactory separation was achieved (Figure 1). Starting the gradient below the melting conditions of one of the four combinations of DNA (two homoduplexes and two heteroduplexes) resulted in reduced or lost separation (data not shown). This is in accordance with previous results obtained for singlecapillary instruments $(2,5)$. Compared to CDCE where the temperature has to be strictly controlled to achieve separation (3), temperature gradient 96-capillary array electrophoresis proved to be very robust. This is based on the fact that during the gradient the capillary will pass through the optimal melting condition of fragment of interest. Accordingly, there is no need for time-consuming optimization procedures once the gradient has been established around the theoretical melting temperature of the fragment.

The sensitivity of the temperature gradient 96-capillary array electrophoresis system for detection of low-frequency mutations in the presence of wild-type was evaluated by mixing mutant and normal alleles in different ratios. Loss of signal from the heteroduplex peaks was observed for mutant fractions below $4 \times 10^{-3}$ (Figure 2). The sensitivity found is in agreement with the sensitivity reported for automated CDCE $(1,2)$. This sensitivity should be sufficient in most areas of mutation and SNP detection/analysis.

Fischer and Lerman (7) first described the effect of single base pair substitution on DNA. Base pair substitution leads to entropy variation and, thus, changes in the DNA stability. The changes are due to a loss or gain of a hydrogen bond and differences in base pair stacking. Melting properties of DNA will reveal peak patterns in the electropherogram that can be used to identify the mutation. Figure 3 displays nine different mutations observed in exon 8 of the TP53 gene. Three of the mutants are from the $\mathrm{CpG}$ site found in codon 273. About $28 \%$ of the mutational events in this exon take place in codon 273. The next largest hot spot is codon 282, with approximately $12 \%$ of the observed mutations in TP53 exon 8. Making a library of known mutations or mutational hot spots in the gene of interest, unknown samples may be verified for their DNA alteration. This would give a fast and accurate method of determining the exact sequence alteration in the sample without having to perform laborious sequencing techniques. This may be achieved with a complete database over all mutations and under the assumption that each mutant has a distinct peak pattern. However, it may be possible for two different mutations to have similar peak pattern. Mixing of two different mutants showing the same peak pattern before analysis to form additional heteroduplexes will identify different mutations (11). Determination of the homoduplexes is

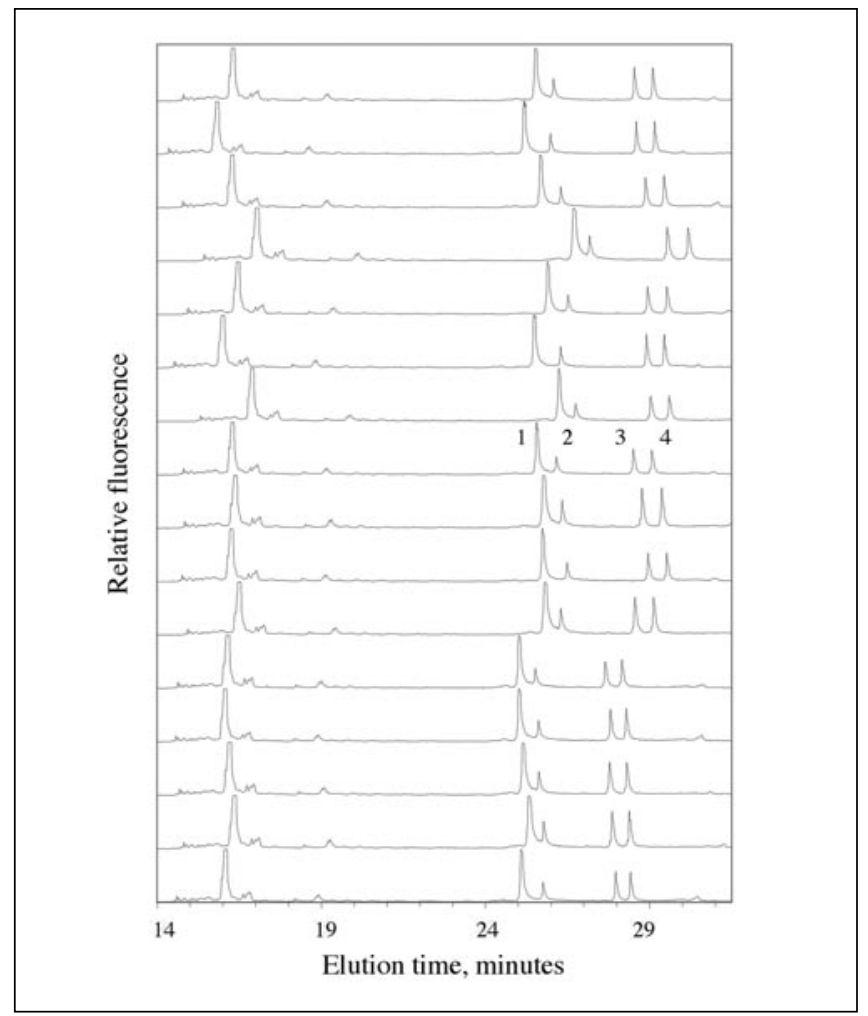

Figure 1. Sixteen representative runs from a 96-capillary parallel analysis of tumor samples. Peaks numbered are wild-type (1), mutant (2), and heteroduplex ( 3 and 4), respectively. Peaks eluting at $16 \mathrm{~min}$ are unincorporated fluorescein-labeled primers. The mutation was a codon 273 CGT $\rightarrow$ TGT transition, verified by DNA sequencing.

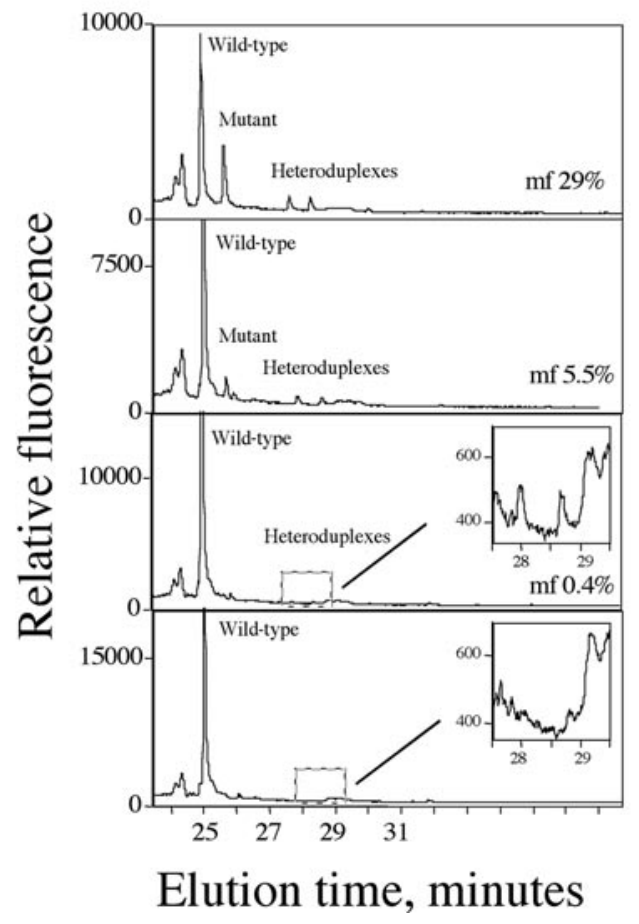

Figure 2. Wild-type sample spiked with a mutant [WiDR cells (ATCC CCL-218), codon 273 CGT $\rightarrow$ TGT] in various ratios. Samples were subjected to temperature gradient 96 -capillary array electrophoresis to obtain separation of homo- and heteroduplexes. All peaks of interest were below the dynamic range of the instrument; thus, measurement of peak area was calculated. Loss of heteroduplex peaks was observed for mutant fractions (mf) below $4 \times 10^{-3}$. 


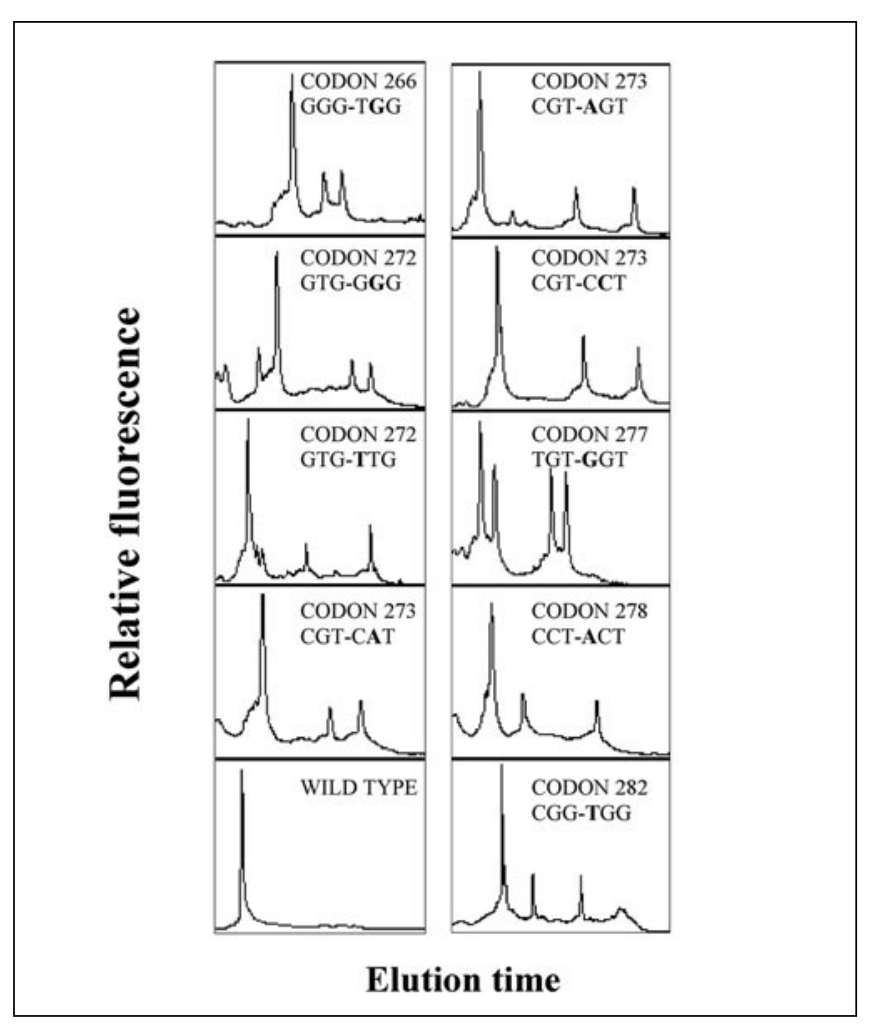

Figure 3. Nine different mutants and one wild-type sample analyzed by temperature gradient 96-capillary array electrophoresis. The mutations are indicated in each electropherogram by codon number followed by canonical and mutated sequence.

accomplished by running a sample together with a wild-type or mutant homoduplex labeled with different fluorescent dye. Standard sequencing instruments have the possibility to point out which peaks of the homoduplexes have the same melting behavior in the capillary. Provisional identification of mutant homoduplexes can be performed by comparing the elution time of a sample peak to that of a co-injected but differentially labeled authentic standard mutant DNA sequences.

Capillary electrophoresis with laser-induced fluorescence detection has moved forward to become a high-throughput method (96 samples/30 min) in mutational research as compared to single column approaches $(2,5,10)$. The standardized commercial sieving matrix, combined with individual temperature compensation through temperature gradient 96-capillary array electrophoresis for each capillary in the instrument, is important for the separation qualities of temperature gradient 96-capillary array electrophoresis. It is also noteworthy that this application can be used on any capillary sequencing instrument with sufficient temperature control in the capillary chamber. The natural and obvious development from 96 capillaries would be to run similar conditions on a 384-capillary instrument, the MegaBACE 4000, opening up a new dimension in mutation analysis.

\section{CONCLUSION}

The results from the present study revealed a fast and sensitive method for the detection of point mutations in exon 8 of the TP53 gene. This method is useful for all DNA iso-melting domains of 100-120 bp, and we are now extending the ap- proach to other exons measuring mutations and SNPs. The main advantage of this method is the simple two-step protocol, PCR followed by electrophoresis.

\section{ACKNOWLEDGMENTS}

This work received financial support by the Thorstensen legacy and The Norwegian Cancer Society.

\section{REFERENCES}

1.Bjørheim, J., P.O. Ekstrøm, E. Fossberg, A.L. Børresen-Dale, and G. Gaudernack. 2001. Automated constant denaturant capillary electrophoresis applied for detection of KRAS exon 1 mutations. BioTechniques 30:972-975.

2.Bjørheim, J., G. Gaudernack, and P.O. Ekstrøm. 2001. Mutation analysis of tp53 exons 5-8 by automated constant denaturant capillary electrophoresis. Tumour Biol. 22:323-327.

3.Bjørheim, J., S. Lystad, A. Lindblom, U. Kressner, S. Westring, S. Wahlberg, G. Lindmark, G. Gaudernack, et al. 1998. Mutation analyses of KRAS exon 1 comparing three different techniques: temporal temperature gradient electrophoresis, constant denaturant capillary electrophoresis and allele specific polymerase chain reaction. Mutat. Res. 403:103-112.

4.Børresen-Dale, A.L., R.A. Lothe, G.I. Meling, P. Hainaut, T.O. Rognum, and E. Skovlund. 1998. TP53 and long-term prognosis in colorectal cancer: mutations in the L3 zinc-binding domain predict poor survival. Clin. Cancer Res. 4:203-210.

5.Ekstrøm, P.O., A.L. Børresen-Dale, H. Qvist, K.E. Giercksky, and W.G. Thilly. 1999. Detection of low-frequency mutations in exon 8 of the TP53 gene by constant denaturant capillary electrophoresis (CDCE). BioTechniques 27:128-134.

6.Ekstrøm, P.O., R. Wasserkort, M. Minarik, F. Foret, and W.G. Thilly. 2000. Two-point fluorescence detection and automated fraction collection applied to constant denaturant capillary electrophoresis. BioTechniques 29:582589.

7.Fischer, S.G. and L.S. Lerman. 1980. Separation of random fragments of DNA according to properties of their sequences. Proc. Natl. Acad. Sci. USA 77:4420-4424.

8.Fischer, S.G. and L.S. Lerman. 1983. DNA fragments differing by single base-pair substitutions are separated in denaturing gradient gels: correspondence with melting theory. Proc. Natl. Acad. Sci. USA 80:1579-1583.

9.Geisler, S., P.E. Lønning, T. Aas, H. Johnsen, O. Fluge, D.F. Haugen, J.R. Lillehaug, L.A. Akslen, and A.L. Børresen-Dale. 2001. Influence of TP53 gene alterations and c-erbB-2 expression on the response to treatment with doxorubicin in locally advanced breast cancer. Cancer Res. 61:2505-2512.

10.Gross, E., M. Kiechle, and N. Arnold. 2001. Mutation analysis of p53 in ovarian tumors by DHPLC. J. Biochem. Biophys. Methods 47:73-81.

11.Guldberg, P. and F. Guttler. 1993. A simple method for identification of point mutations using denaturing gradient gel electrophoresis. Nucleic Acids Res. 21:2261-2262.

12.Hainaut, P., T. Hernandez, A. Robinson, P. Rodriguez-Tome, T. Flores, M. Hollstein, C.C. Harris, and R.Montesano. 1998. IARC Database of p53 gene mutations in human tumors and cell lines: updated compilation, revised formats and new visualisation tools. Nucleic Acids Res. 26:205-213.

13.Hovig, E., B. Smith-Sørensen, A. Brøgger, and A.L. Børresen. 1991. Constant denaturant gel electrophoresis, a modification of denaturing gradient gel electrophoresis, in mutation detection. Mutat. Res. 262:63-71.

14.Khrapko, K., J.S. Hanekamp, W.G. Thilly, A. Belenkii, F. Foret, and B.L. Karger. 1994. Constant denaturant capillary electrophoresis (CDCE): a high resolution approach to mutational analysis. Nucleic Acids Res. 22:364-369.

15.Somasundaram, K. 2000. Tumor suppressor p53: regulation and function. Front. Biosci. 5:D424-D437.

Address correspondence to Dr. Per O. Ekstrøm, Department of Surgical Oncology, Institute for Cancer Research, The Norwegian Radium Hospital, Oslo, Norway. e-mail: peroe@ radium.uio.no contact Reprints@BioTechniques.com 